\title{
Relationship Between Perceived Risk and Physician Recommendation and Repeat Mammography in the Female Population in Tehran, Iran
}

\author{
Mahdi Moshki ${ }^{1}$, Parvaneh Taymoori ${ }^{2 *}$, Sahmireh Khodamoradi ${ }^{3}$, Daem Roshani ${ }^{2}$
}

\begin{abstract}
Iranian women are at high risk of low compliance with repeat mammography due to a lack of awareness about breast cancer, negative previous experiences, cultural beliefs, and no regular visits to a physician. Thus research is needed to explore factors associated with repeated mammography participation. Applying the concept of perceived risk as the guiding model, this study aimed to test the fit and strength of the relationship between perceived risk and physician recommendation in explaining repeat mammography. A total of 601 women, aged 50 years and older referred to mammography centers in region 6 , were recruited via a convenience sampling method. Using path analysis, family history of breast cancer and other types of cancer were modeled as antecedent perceived risk, and physician recommendation and knowledge were modeled as an antecedent of the number of mammography visits. The model explained $49 \%$ of the variance in repeat mammography. The two factors of physician recommendation and breast selfexamination had significant direct effects $(\mathrm{P}<0.05)$ on repeat mammography. Perceived risk, knowledge, and family history of breast cancer had significant indirect effects on repeat mammography through physician recommendation. The results of this study provide a background for further research and interventions not only on Iranian women but also on similar cultural groups and immigrants who have been neglected to date in the mammography literature.
\end{abstract}

Keywords: Perceived rRisk - physician recommendation - mammography repeat - breast cancer

Asian Pac J Cancer Prev, 17, Cancer Control in Western Asia Special Issue, 161-166

\section{Introduction}

Mammography screening is believed to reduce breast cancer mortality by 25\%-35\% (Yoo et al., 2013). The implementation rate of regular mammography screening programs in developed countries varies between $46 \%$ and $72 \%$. Due to the absence of a national breast cancer screening program in Iran, no official statistics have been published about the exact mammography rates throughout the country (Harirchi I et al., 2011). However, Taymoori et al. reported only $5.7 \%$ of women over 40 years old to regularly take mammograms (Taymoori et al., 2012b). Another study on 400 Iranian women in the same age group found an even lower rate (i.e. 3.7\%) (Abedian et al., 2006).

Theories aiming at promoting favorable health behaviors (e.g. regular mammography) among women generally incorporate the concept of perceived risk (or related constructs) into their framework (rosenstock I, 1974). Perceived risk of breast cancer can be defined as a woman's perception of her chance of developing the disease. Research on the relationship between perceived risk and taking mammograms has yielded controversial results. The risk perception was significantly associated with colon cancer screening behavior (Kim et al., 2008). The similar results did not show significant relationship between risk perception and mammography. It is also indicated that women who believed their susceptibility were high were less likely to be screened; women with higher perceived susceptibility had a lower tendency toward screening (Facione, 2002; Calvocoressi et al., 2004). Finally, a meta-analytic review found that women who perceived a higher breast cancer risk were more likely to have mammograms although the association was weak (Katapodi et al., 2004). Moreover, higher perceived susceptibility to breast cancer was introduced as a predictor of clinical breast examination and frequent physician visits among Malaysian women (Parsa and Kandiah, 2010).

The family history of breast cancer can be considered as a determinant of the relationship between perceived risk and regular mammography. In fact, studies on women with a family history of breast cancer have reported a positive relationship between higher perceived risk and adherence to mammography guidelines (Walker et al., 2013). Based on available literature, women believe that a positive family history increases their risk of developing breast cancer (Buxton et al., 2003; Silk et al., 2006; Caruso et

${ }^{1}$ Department of Public Health, School of Health Sciences, Social Development \& Health Promotion Research Center, Gonabad University of Medical Sciences, Gonabad, ${ }^{2}$ Social Determinants of Health Research Center, ${ }^{3}$ Student Research Committee, Kurdistan University of Medical Sciences, Sanandaj, Iran. *For correspondence: parvaneh_tay@yahoo.com 
al., 2009). Their perception of risk is, hence, formed based on their family history of breast cancer. Nevertheless, since about $80 \%$ of cases of breast cancer do not run in families, considering family history as the only risk factor in determining one's risk of breast cancer will lead to an inaccurate perception of risk and affect regular mammography rates.

The observed inconsistencies in the results of previous studies might also be justified by the role of women's perception of breast cancer in their adherence to the provided mammography guidelines. Molina suggested the perceived risk of breast cancer to have a moderating effect on the relationship between recommendations to take mammograms and women's tendency toward regular mammography (Molina et al., 2014). It is identified that physician recommendations are the only moderator of the association between perceived risk of breast cancer and regular mammography (Haber et al., 2012). Due to the significance of interpersonal relationships in the Iranian culture, recommendations made by physicians can encourage Iranian women to take regular mammograms (Miok C and 2000). Likewise, a study on Malaysian women reported physicians' recommendations as a major predictor of obtaining mammograms and performing breast self-examination (BSE) (Dunn et al., 2010).

Unfortunately, the determinants of Iranian women's willingness to participate in regular mammography screening is not well understood. In fact, few studies have examined the needs of Iranian women who refuse to take regular mammograms despite having a history of mammography (Babu et al., 2011; Taymoori et al., 2015 ). Meanwhile, although many studies have highlighted the role of perceived risk and physician recommendation in Iranian women's adherence to mammography guidelines (Ahmadian et al., 2011; Farhadifar et al., 2016), most of these studies have neglected the interaction effects of the mentioned factors. Empirical evaluation of such interaction effects would not only enhance the existing knowledge about the psychosocial determinants of mammography screening, but also facilitates the identification of the best target groups for relevant interventions. Hence, the present study sought to determine the strength of the relationships of regular mammography with perceived risk and physician recommendation among women in Tehran, Iran. The relationships between repeated mammography, breast self exam (BSE) and clinical breast exam (CBE) are also investigated.

\section{Materials and Methods}

This cross-sectional study used convenience sampling to select 708 women over 50 years old who referred to public and private mammography centers in district 6 from 22 regions in Teheran, Iran. Of all the participants, 48 subjects were not eligible and were thus excluded. Furthermore, 59 other women were also excluded due to missing data. The final sample size was hence 601 women and the response rate was $93 \%$. Women were included if they aged $\geq 50$ years, were literate, and had a history of repeated mammography (i.e. two or more mammograms) in the past five years. Participants with breast cancer, as
Table1. Demographic Characteristics ( $N=601)$

\begin{tabular}{lllll}
\hline Variables & $\mathrm{N}$ & $\%$ & Mean & SD \\
\hline Age & & & 59.0 & 6.4 \\
Marital status & & & &
\end{tabular}

$\begin{array}{lll}\text { Single } & 189.0 & 31.5 \\ \text { Married } & 411.0 & 68.5\end{array}$

Educational Status

$\begin{array}{lll}\text { Academic } & 276.0 & 46.0 \\ \text { Non Academic } & 324.0 & 54.0 \\ \text { Employment Status } & & \\ \text { employed } & 317.0 & 52.8 \\ \text { employed } & 283.0 & 47.2\end{array}$

Insurance Status

$\begin{array}{lll}\text { Yes } & 507.0 & 84.5 \\ \text { No } & 93.0 & 15.5\end{array}$

Breast cancer family history

$\begin{array}{lll}\text { Yes } & 49.0 & 8.2 \\ \text { No } & 551.0 & 91.8\end{array}$

Other cancer family history

$\begin{array}{lll}\text { Yes } & 86.0 & 14.4 \\ \text { No } & 514.0 & 85.4\end{array}$

Breast Self-

Examination(BSE)

$\begin{array}{lll}\text { Regular } & 90.0 & 15.0 \\ \text { Irregular } & 418.0 & 69.4 \\ \text { Not doing } & 93.0 & 15.6\end{array}$

Clinical Breast Examination (CBE)

$\begin{array}{lll}\text { Regular CBE } & 177.0 & 29.5 \\ \text { Irregular CBE } & 328 & 54.5 \\ \text { lack of CBE } & 96.0 & 20.0\end{array}$

Knowledge of the interval mammography

$\begin{array}{lll}\text { Annual } & 207.0 & 34.5 \\ \text { 2-3 years } & 152.0 & 25.3 \\ \text { 3 years }> & 184.0 & 30.7 \\ \text { Don't know } & 57.0 & 9.5\end{array}$

Perceived threat

Number of repeat mammogram

\begin{tabular}{cll}
1 & 228.0 & 38.0 \\
2 & 110.0 & 18.3 \\
3 & 177.0 & 29.5 \\
4 & 48.0 & 8.0 \\
$\geq 5$ & 37.0 & 6.2 \\
Physician recommenda- & & \\
tion & & \\
Yes & 522.0 & 87.0 \\
No & 138.0 & 23.0 \\
\hline
\end{tabular}


Table 2. Correlation Coefficients Matrix of Variables $(\mathrm{N}=601)$

\begin{tabular}{|c|c|c|c|c|c|c|c|}
\hline Variables & 2 & 3 & 4 & 5 & 6 & 7 & 8 \\
\hline 1. Perceived threat & $0.5^{*}$ & $0.2^{*}$ & $0.2 *$ & $0.3^{*}$ & $0.3^{*}$ & $0.3 *$ & $0.3^{*}$ \\
\hline 2. Knowledge & & $0.2^{*}$ & 0.1 & $0.2^{*}$ & $0.2^{*}$ & $0.2 *$ & $0.4^{*}$ \\
\hline 3. Family History of Breast Cancer & & & $0.4^{*}$ & $0.2^{*}$ & $0.1^{*}$ & $0.3 *$ & 0.1 \\
\hline 4. Family History of Other Cancer & & & & $0.2^{*}$ & $0.2^{*}$ & 0.2 & 0.1 \\
\hline 5. BSE & & & & & $0.5^{*}$ & $0.4^{*}$ & $0.3^{*}$ \\
\hline 6. Physician Recommendation & & & & & & $0.4^{*}$ & $0.7^{*}$ \\
\hline 7. $\mathrm{CBE}$ & & & & & & & $0.3^{*}$ \\
\hline 8. Number of Mammography & & & & & & & \\
\hline
\end{tabular}

BSE Breast Self-Examination; CBE Clinical Breast Examination; ${ }^{*} \boldsymbol{P}<0.05$ (two tailed); ${ }^{* *} \boldsymbol{P}<0.01$

well as pregnant or lactating women, were excluded. The Ethical committee of Kurdistan Medical University approved the study. The participants were informed about the aim of the study and consent form was obtained from all participants.

The participants were asked to complete a questionnaire containing demographic characteristics (age, marital status, education level, insurance coverage, and family history of breast cancer and other types of cancer). Women's performances in BSE were categorized as regular BSE, irregular BSE, and no BSE. The frequency of $\mathrm{CBE}$ was recorded as annual $\mathrm{CBE}$, irregular $\mathrm{CBE}$ once every several years, and no CBE. Knowledge about the time interval of mammography in women was determined though choosing one of the three following options: once a year, once every two to three years, and once more than every three years. These questions were re-coded into dichotomous variables by coding false, I do not know $=0$ and true $=1$. Ten items, extracted from Champion's Health Belief Model Scale (Champion, 1993; Champion, 1999) were used to measure perceived risk. The items were scored on a five-point Likert scale from one (strongly disagree) to five (strongly agree). The items were validated and revised by two experts in health education and health promotion from Kurdistan University of Medical Sciences. In order to determine the test-retest reliability of the questionnaire, it was administered among 50 randomly selected individuals and re-administered with a two-week interval $(r=0.8)$. Physician recommendation for mammography was evaluated by raising the following yes/no question: "Has a doctor recommended you to take a mammogram during the past year?"

\section{Data Analysis}

The SPSS version 16 was used for descriptive statistics and correlation detection among the variables. Also, LISREL 8.8 was used to analyze the data via the path analysis method with maximum likelihood estimation and a covariance matrix, Root Mean Squared Error of Approximation (RMSEA) lower than 0.1, adjusted Goodness of Fit Index (GFI) higher than 0.8.

In addition, simultaneous investigation of the relationship between the variables of the study and path analysis showed direct, indirect, and general effects of variables on the outcome variable. Specifically, in accordance with previous findings, family history of breast cancer and any other cancer were modeled as an antecedent perceived risk (Khalili and Shahnazi, 2010; Haber et al., 2012), BSE and CBE (Myers et al., 2008), while physicians' recommendation and knowledge were modeled as an antecedent of number of mammography (Babu et al., 2011; Ahmadian et al., 2012a). The BSE, and CBE were subsequently modeled as additional antecedents of mammography repeat. Indicators of each variable were fixed to 1.0 in order to create a metric scale. Further, all variables were fixed to 0 error, which is commensurate with OLS regression analyses path models. Variables considered to be covariates, and included in all analyses, were age, education, geographic region, and income. Age, education, and income were mean centered for ease of interpretation.

\section{Results}

The women's mean age was 58.9 years (SD, 6.4 years), the majority of participants reported irregular BSE (69.4 $\%$ ). In terms of CBE, only $29.5 \%$ had regular CBE. The biggest proportion of repeat mammogram (38\%) repeated their mammograms one time. Table 1 gives further details on the demographic characteristics of the participants. Table 2 shows Bivariate correlations for all variables of interest. The variable of physicians' recommendation had the highest correlation $(r=0.7)$ with the number of mammography followed by knowing time interval for mammography $(r=0.4)$ and $\mathrm{CBE}(r=0.3)$.

The results of the model resulted in a moderate fit of the data $\left[X^{2}(8)=38.6 ; P<0.01 ; \mathrm{GFI}=.95 ; \mathrm{RMSEA}=.09\right]$ via conventional cut-off criteria and given the intricacy of the model [42]. Still, freeing the direct paths for the variables perceived risk, knowledge, family history of breast cancer and any other types of cancer on CBE improved fit and explained an additional $3 \%$ of the variance $\left(X^{2}(4)=35.2 ; P<0.01\right)$. The addition of the direct paths of the risk perception, knowledge, family history of breast cancer and any of cancer variables on the number of mammography, however, did not add to the overall fit $(P>.05)$ and thus were dropped. The final model $\left[X^{2}(4)=8.5 ; \mathrm{p}=0.3 ; \mathrm{CFI}=1.0 ; \mathrm{RMSEA}=0.02\right]$ is presented in Figure 1 and suggested a good fit of these data (Baron and Kenny, 1986).

Overall, the model explained $49 \%$ of the variance in the number of mammography. The two significant $(\mathrm{p}<.05)$ direct effects were from physicians' recommendation (standardized effect $=0.6$; unstandardized 


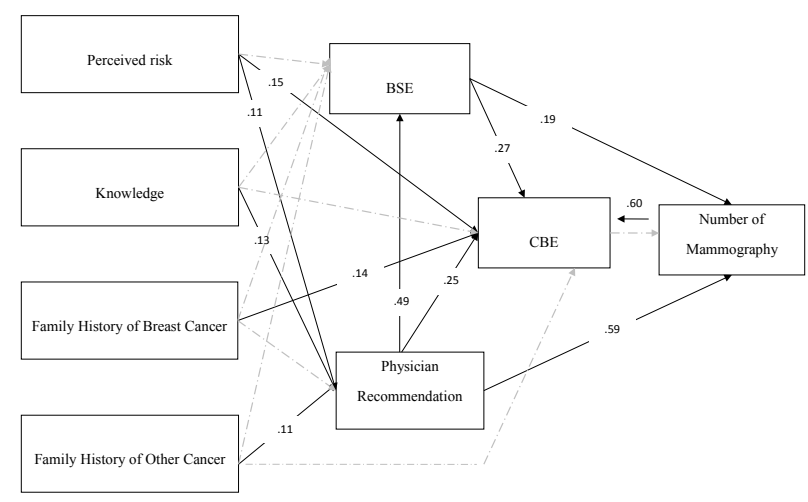

Figure 1.The Final Model of Breast Cancer

effect $=80.1, \mathrm{SE}=5.7)$ and BSE (standardized effect $=0.2$; unstandardized effect $=28.1, \mathrm{SE}=8.5$ ); $\mathrm{CBE}$ did not have an independent effect, through physicians' recommendation (standardized effect $=0.25$; unstandardized effect $=$ $0.2, \mathrm{SE}=0.1$ ) and BSE (standardized effect $=0.3$; unstandardized effect $=0.2, \mathrm{SE}=0.04)$ explained $38 \%$ of its variance. Further, risk perception had significant indirect effects on mammography repeat through physician recommendation (standardized effect $=0.1$; unstandardized effect $=17.3,95 \% \mathrm{CI}=2.8$ to 31.8 ), knowledge (standardized effect $=0.1$; unstandardized effect $=12.3,95 \% \mathrm{CI}=3.2$ to 21.3 ) and breast cancer family history (standardized effect $=0.1$; unstandardized effect $=10.5,95 \% \mathrm{CI}=2.4$ to 18.5 ). Considering physicians' recommendation, it had a significant effect on BSE (standardized effect $=0.5$; unstandardized effect $=0.4, \mathrm{SE}=0.03$ ), and explained $34 \%$ of its variance. Subsequently, physicians' recommendation had $8 \%$ of its variance explained by perceived risk (standardized effect $=0.1$; unstandardized effect $=0.2, \mathrm{SE}=0.1$ ), knowledge (standardized effect $=.13$; unstandardized effect $=0.1, \mathrm{SE}$ $=0.1$ ), and family history of breast cancer (standardized effect $=0.1$; unstandardized effect $=0.1, \mathrm{SE}=0.04$ ).

The interactive effects of physician recommendation and BSE on mammography repeat were tested with a regression model, which were also controlled for CBE. Prior to the analysis, the variables of the study were centered before computing interaction terms as recommended by Aiken and West (Hu and Bentler, 1999). The regression model showed a significant interaction effect .

\section{Discussion}

In this study, only $15 \%$ of the participants reported that they practiced BSE on a regular (monthly) basis. The breast cancer practices rate among the Iranian women were low. Women performed less BSE regularly than other women in the world (Fouladi et al., 2013; Naghibi et al., 2015). Therefore, delineating the cognitive correlates of breast cancer preventive behaviors is essential to the development of effective interventions.

About $70 \%$ of women did not have true knowledge of mammogram yearly intervals. This finding is in line with the findings of studies by Tolma and Rakowski (Rakowski et al., 2006; Tolma et al., 2011) which reported knowledge of mammography time intervals had a direct and significant impact on increased rate of repeat mammography. Women's lack of knowledge has been reported as one of the factors which prevent women taking measures such as mammography for early detection of breast cancer (Harris et al., 2003; Montazeri et al., 2008; Khanjani $\mathrm{N}$ et al., 2012).

The results of this cross-sectional study showed that the model fitted the data; in addition, physicians' recommendation and BSE were directly associated with mammography repeat behavior among Iranian women.

The findings revealed that the model could explain $49 \%$ of the variance. The findings regarding physician recommendation is consistent with the results of previous studies that found the moderating effect of perceived risk of breast cancer on the relationship between physicians' recommendations and mammography intentions (Molina et al., 2014). However, in Haber's study, the relationship between perceived risk and physician recommendation was not found (Haber et al., 2012). Further, the results of our cross-sectional study should be interpreted with caution, as the increased number of mammography might result in more frequent physicians' recommendations, an idea inherent in the reciprocal nature of these variables (Meissner et al., 2007; Ahmadian et al., 2011; Ahmadian et al., 2012b). The finding that BSE had a direct path with mammography proposes that having regular BSE may be effective determinants for women in this cultural context. However, the relationship found between physicians' recommendation and BSE indicates that physician recommendation can increase BSE. Furthermore, we conducted a test to investigate the relationship between physicians' recommendation and BSE, and the results showed that the two variables had a mutual interaction, which affected the frequency of mammography among the studied sample. This finding is not consistent with the results reported by other researchers, as they did not observe such an interaction (Jelinski et al., 2005). In addition, the observed interaction must also be investigated among older women, as it may have more significant effects on older women than younger women; however, we did not find a similar relationship. The interaction between these two variables is an interesting filed of research in mammography literature that has received relatively little attention; hence, further studies should examine how these two variables interact.

An important contribution of this research was the investigation of the effect of perceived risk on mammography repeat. Contrary to our hypothesis, perceived risk had no direct effect on mammography behavior. In some studies, the researchers found a weak positive association between higher perceived risk and adherence to mammography (Katapodi et al., 2004; Walker et al., 2013). This result is also contrary to the findings of other researchers who reported the effect of perceived risk on mammography behavior (Taymoori et al., 2013; Taymoori et al., 2015 ). However, in our study, perceived risk, knowledge, and family history of breast cancer and other types of cancer were indirectly associated with behavior by physician recommendation. Further research should try to verify these results via studying women from different ethnicities. Our findings provided support for the 
value of perceived risk as a means of indirect promotion of mammography behavior via increasing physicians' recommendation. This is in line with the findings of other researches (Parsa and Kandiah, 2010; Farhadifar et al., 2016). However, future research should test the same relationships in longitudinal studies. Enhancing risk perceptions might be utilized as an important method for encouraging Iranian women to undergo mammography, because risk perception is a prerequisite for mammography behavior. This is an important solution for this population because there are unique cultural challenges that make it even more difficult for women to perform mammography repeat. We found both direct and indirect effects of physicians' recommendation on breast cancer screening behaviors, and our findings are consistent with the results of some previous studies (Moodi et al., 2011; Ahmadian et al., 2012b). This finding is particularly more significant for Iranian women who follow the Iranian culture, because people in Iran usually follow the recommendation of physician more strictly (Lamyian et al., 2007; Babu et al., 2011). Thus, the physician's recommendation can enhance mammography behavior.

According to the results of this model, the family history of breast cancer was not a significant predictor of mammogram repeat. The findings from other researches have been mixed that may influenced by measurement of risk estimate breast cancer. Tracy found family history had positive effects on mammography or BSE (Montazeri et al., 2008; Tracy et al., 2008). Others have not found a relationship between family history of breast cancer and mammogram (West et al., 2003)

In this study, CBE did not have a direct effect on mammography after controlling physicians' recommendation. This is consistent with the results of a recent research on mammography behavior (Saslow et al., 2004). As modeled in our study, the bivariate relationships between CBE, physicians' recommendation, and mammography suggested that CBE might be best considered as an antecedent, and not a consequence, of physicians' recommendation.

\section{Limitations}

The present study had several limitations. First, the data collected via a self-report questionnaire. As another limitation, this study conducted as a cross-sectional study, which makes it impossible to make a conclusion about antecedents of successful mammography behavior change. Women may make behavioral choices during the pre-menopause / menopause period that contribute to lifelong behavioral patterns. However, longitudinal studies are needed to evaluate the changes in behaviors. The findings of the study might be possibly influenced by socio-economic status of the studied participants (who had referred to mammography centers in region 6, Tehran). One of the other limitations of this study was the difference between the scales used in the current study and other studies to measure the risk perception. It made it difficult to assess our results and compare them with the results of previous studies. In addition, the results might be affected by lack of a ranking system for different levels of risk perception and the subjective measurement of this variable. Utilization of tools which both subjectively and objectively measure risk perception (like the Gail model) (McCarthy et al., 2015) can help to clarify the interaction between risk perception and family history of breast cancer and how they affect repeat mammography. Finally, we did not consider the number of close family members with breast cancer while this factor may also affect the perception of the risk of breast cancer.

The rate of mammography repeat among Iranian women is low. The findings of this study strongly support the relationship between physician recommendation and undergoing mammography repeat among Iranian women. Further, the possible influence of physician recommendations to obtain mammogram should be considered when interventions are developed to enhance mammography behavior. For example, sending reminder cards and utilization of different communication tools can be considered as some possible interventions. The efficacy of these methods should be evaluated in future research. Highlighting the need for recommendations from physicians is becoming important for older women because they are most often no longer in the care of a physician/gynecologist. It is necessary to design special measures and interventions for this population because, due to the unique cultural challenges, mammography repeat is even more difficult for them.

\section{Acknowledgments}

This article is extracted from Master Thesis Sahmireh Khodamoradi, as "assessment of the physician recommendation and perceived risk with repeated mammography in the female population in Tehran, Iran. We would like to thank Deputy of Research and Technology of Kurdistan University of Medical Sciences for providing financial support for this study.

\section{References}

Abedian K, Shah Hosseini Z, Adeli M (2006). Survey of health beliefs of women about performing mammography in the health servic centers of Sari. J Mazandaran Univ Med Sci, 16, 90-8.

Ahmadian M, Samah AA, Redzuan Mr, et al (2011). Barriers to mammography among women attending gynecologic outpatient clinics in Tehran, Iran. Sci Res Essays, 6, 5803-11.

Ahmadian M, Samah AA, Redzuan Mr, et al (2012a). Participation in breast cancer prevention: Assessing women's knowledge and their participation in mammography in Tehran, Iran. Sci Res Essays, 7, 915-22.

Ahmadian M, Samah AA, Redzuan Mr, et al (2012b). Predictors of mammography screening among Iranian women attending outpatient clinics in Tehran, Iran. Asian Pac J Cancer Prev, 13, 969-74.

Babu GR, Samari G, Cohen SP, et al (2011). Breast cancer screening among females in Iran and recommendations for improved practice: a review. Asian Pac J Cancer Prev, 12, 1647-55.

Baron RM, Kenny DA (1986). The moderator-mediator variable distinction in social psychological research: Conceptual, strategic, and statistical considerations. J Pers Soc Psychol, 51, 1173-82.

Buxton JA, Bottorff JL, Balneaves LG, et al (2003). Women's 
perceptions of breast cancer risk: Are they accurate?. Can J Public Health, 94, 422-6.

Calvocoressi L, Kasl SV, Lee CH, et al (2004). A prospective study of perceived susceptibility to breast cancer and nonadherence to mammography screening guidelines in African American and White women ages 40 to 79 years. Cancer Epidemiol Biomarkers Prev, 13, 2096-105.

Caruso A, Vigna C, Marozzo B, et al (2009). Subjective versus objective risk in genetic counseling for hereditary breast and/ or ovarian cancers. J Exp Clin Cancer Res, 28, 157.

Champion VL (1993). Instrument refinement for breast cancer screening behaviors. Nurs Res, 42, 139-43.

Champion VL (1999). Revised susceptibility, benefits, and barriers scale for mammography screening. Nurs Res, $\mathbf{2 2}$, 341-8.

Dunn RA, Tan A, Samad I (2010). Does performance of breast self-exams increase the probability of using mammography: evidence from Malaysia. Asian Pac J Cancer Prev, 11, 417-21.

Facione NC (2002). Perceived risk of breast cancer. Cancer Pract, 10, 256-61.

Farhadifar F, Yamile M, Taymoori P, et al (2016). Mediators of repeat mammography in two tailored interventions for Iranian women. BMC Public Health, 16, 149.

Fouladi N, Pourfarzi F, Mazaheri E, et al (2013). Beliefs and behaviors of breast cancer screening in women referring to health care centers in northwest Iran according to the champion health belief model scale. Asian Pac J Cancer Prev, 14, 6857-62.

Haber G, Ahmed NU, Pekovic V (2012). Family history of cancer and its association with breast cancer risk perception and repeat mammography. Am J Public Health, 102, 2322-9.

Harirchi I, Kolahdoozan S, Karbakhsh M, et al (2011). Twenty years of breast cancer in Iran: downstaging without a formal screening program. Ann Oncol, 22, 93-7.

Harris DM, Miller JE, Davis DM (2003). Racial differences in breast cancer screening, knowledge and compliance. $J$ Natl Med Assoc, 95, 693-701.

$\mathrm{Hu}$ Lt, Bentler PM (1999). Cutoff criteria for fit indexes in covariance structure analysis: Conventional criteria versus new alternatives. Struct Equ Modeling, 6, 1-55.

Jelinski SE, Maxwell CJ, Onysko J, et al (2005). The influence of breast self-examination on subsequent mammography participation. Am J Public Health, 95, 506-11.

Katapodi MC, Lee KA, Facione NC, et al (2004). Predictors of perceived breast cancer risk and the relation between perceived risk and breast cancer screening: a meta-analytic review. Prev Med, 38, 388-402.

Khalili AF, Shahnazi M (2010). Breast cancer screening (breast self-examination, clinical breast exam, and mammography) in women referred to health centers in Tabriz, Iran. Indian J Med Sci, 64, 149-62.

Khanjani N, Noori A, Rostami F (2012). The knowledge and practice of breast cancer screening among women in Kerman, Iran. Al-Ameen J Med Sci , 5, 221-6.

Kim SE, Pérez-Stable EJ, Wong S, et al (2008). Association between cancer risk perception and screening behavior among diverse women. Arch Intern Med, 168, 728-34.

Lamyian M, Hydarnia A, Ahmadi F, et al (2007). Barriers to and factors facilitating breast cancer screening among Iranian women: a qualitative study. East Mediterr Health $J$, 13, 1160-9.

McCarthy A, Keller B, Kontos D, et al (2015). The use of the Gail model, body mass index and SNPs to predict breast cancer among women with abnormal (BI-RADS 4) mammograms. Breast Cancer Res, 17, 1.

Meissner HI, Breen N, Taubman ML, et al (2007). Which women aren't getting mammograms and why?(United States). Cancer Causes Control, 18, 61-70.

Miok C, (2000). Leeknowledge, "Barriers and motivators related to cervical cancer screening among Karean-American Women cancer Nursing". Cancer Nurs, 23, 168-75.

Molina Y, Thompson B, Ceballos RM (2014). Physician and family recommendations to obtain a mammogram and mammography intentions: The moderating effects of perceived seriousness and risk of breast cancer. $J$ Womens Health Care, 3, 199-206.

Montazeri A, Vahdaninia M, Harirchi I, et al (2008). Breast cancer in Iran: need for greater women awareness of warning signs and effective screening methods. Asia Pac Fam Med, 7, 6-9.

Moodi M, Mood MB, Sharifirad GR, et al (2011). Evaluation of breast self-examination program using Health Belief Model in female students. J Res Med Sci, 16, 316.

Myers CD, Jacobsen PB, Huang Y, et al (2008). Familial and perceived risk of breast cancer in relation to use of complementary medicine. Cancer Epidemiol Biomarkers Prev, 17, 1527-34.

Naghibi SA, Shojaizadeh D, Yazdani Cherati J, et al (2015). Breast cancer preventive behaviors among Iranian women. Acta Med Iranica, 53, 62-68.

Parsa P, Kandiah M (2010). Predictors of adherence to clinical breast examination and mammography screening among Malaysian women. Asian Pac J Cancer Prev, 11, 681-8.

Rakowski W, Meissner H, Vernon SW, et al (2006). Correlates of Repeat and Recent Mammography for Women Ages 45 to 75 in the 2002 to 2003 Health Information National Trends Survey (HINTS 2003). Cancer Epidemiol Biomarkers Prev, 15, 2093-101.

Rosenstock I (1974). The health belief model and preventive behavior. Health Educ Monogr, 4, 354-86.

Saslow D, Hannan J, Osuch J, et al (2004). Clinical breast examination: practical recommendations for optimizing performance and reporting. CA Cancer J Clin, 54, 327-44.

Silk KJ, Bigbsy E, Volkman J, et al (2006). Formative research on adolescent and adult perceptions of risk factors for breast cancer. Soc Sci Med, 63, 3124-36.

Taymoori P, Molina Y, Roshani D (2015 ). Effects of a randomized controlled trial to increase repeat mammography screening in Iranian women. Cancer Nurs, 38, 51-60.

Taymoori P, Moshki M, Roshani D (2013). Facilitator psychological constructs for mammography screening among Iranian women. Asian Pac J Cancer Prev, 15, 7309-16.

Tolma EL, Stoner JA, Li J, et al (2011). Predictors of regular mammography use among American Indian women in Oklahoma: a systematic review. Hereditary Cancer Clin Pract, 9, 2-9.

Tracy KA, Quillin JM, Wilson DB, et al (2008). The impact of family history of breast cancer and cancer death on women's mammography practices and beliefs. Genet Med, 10, 621-5.

Walker MJ, Chiarelli AM, Knight JA, et al (2013). Perceived risk and adherence to breast cancer screening guidelines among women with a familial history of breast cancer: a review of the literature. Breast J, 22, 395-404.

West DS, Greene PG, Kratt PP, et al (2003). The impact of a family history of breast cancer on screening practices and attitudes in low-income, rural, African American women. $J$ Womens Health, 12, 779-87.

Yoo KB, Kwon JA, Cho E, et al (2013). Is mammography for breast cancer screening cost-effective in both Western and asian countries?: results of a systematic review. Asian Pac J Cancer Prev, 14, 4141-9. 